(C) 2012 IEEE. Personal use of this material is permitted. Permission from IEEE must be obtained for all other uses, in any current or future media, including reprinting/republishing this material for advertising or promotional purposes, creating new collective works, for resale or redistribution to servers or lists, or reuse of any copyrighted component of this work in other works.

This is the accepted version of a paper presented at the $31^{\text {st }}$ IEEE/AIAA Digital Avionics Systems Conference (DASC) in October 2012. The published version can be found in IEEE Xplore:

http://ieeexplore.ieee.org/xpl/articleDetails.jsp?tp=\&arnumber=6382368 


\title{
COMPARISON OF COORDINATED AND UNCOORDINATED PHY/MAC SCHEMES FOR BEACONING
}

\author{
Nico Franzen \\ German Aerospace Center (DLR), Wessling, Germany
}

\begin{abstract}
Beaconing is an important technique for the broadcast of surveillance information, e.g., between aircraft, as well as for the exchange of neighborhood information in mobile ad-hoc networks. For aeronautical communication and surveillance, the scarcity of available spectrum is an important issue. Therefore, this paper compares the spectral efficiency of two advanced beaconing schemes to plain Aloha. The first advanced method uses coordinated medium access within self-organized network cells. The second option is uncoordinated, like plain Aloha, but uses strong error correction coding and successive interference cancellation to decode multiple overlapping messages. The results show that for aeronautical beaconing, both advanced solutions promise a much higher efficiency than plain Aloha. Under optimistic assumptions, the coordinated cellular approach could be somewhat ahead of the advanced Aloha scheme. Yet, for safety-critical aeronautical applications, the advantage may be seen as too small to outweigh drawbacks of the coordinated approach like its dependence on, e.g., accurate time synchronization between users.
\end{abstract}

\section{Introduction}

The broadcast of small messages from one network node to all others in vicinity is known as beaconing. Today, surveillance beaconing exists in many inter-vehicular scenarios, where the messages announce a node's position, speed, and other data to increase situational awareness, and hence increase safety. According to [1], aeronautical surveillance beaconing traffic will increase significantly in the future. What's more, mobile ad-hoc networks (MANETs) like the one described in [2] require extensive knowledge at each network node (aircraft) about all others in range. The exchange of this information is another application for beaconing. To date, there are two systems predominantly in use for the aeronautical Automatic Dependent Surveillance Broadcast (ADS-B) application. These are the Universal Access Transceiver (UAT), and the
Secondary Surveillance Radar (SSR) mode S extended squitter. Both use plain Aloha, i.e., random and uncoordinated medium access control (MAC), without any special measures on the physical layer (PHY) to mitigate interference when messages collide [3]. In this paper, an upper bound on the spectral efficiency of such plain Aloha beaconing is used as a benchmark for two advanced beaconing schemes in the aeronautical scenario. By spectral efficiency, we mean the aggregated data rate per bandwidth at which a node can receive useful information from all other nodes within a given communication range. This quantity is proportional to the user density that can be supported per bandwidth. Hence, it should be as large as possible to avoid wasting spectrum in the bands reserved for aeronautical communication and surveillance, which are already congested today.

The first advanced beaconing scheme is the coordinated approach lined out in [4], which is an extension of [5]. It divides the world into a regular pattern of cells and assigns each cell a communication channel based on a reuse scheme. The users within each cell share this channel by means of a self-organizing Time-Division MultipleAccess (TDMA) method with variable guard times. The second advanced beaconing method is the completely uncoordinated option described in [6]. This scheme uses plain Aloha MAC, but employs strong error correcting codes and successive interference cancellation on the PHY layer to receive multiple overlapping messages correctly. It has therefore been termed Advanced Aloha.

This paper makes two main contributions. At first, the derivation of the spectral efficiency bound for plain Aloha under the special constraints of beaconing, to be explained in detail in Chapter 2 . Secondly, the analysis of the spectral efficiency of the coordinated beaconing scheme, which is not contained in [4]. This allows us to take existing results for Advanced Aloha and compare both advanced solutions to plain Aloha. 
The rest of the paper is structured as follows. Chapter 2 explains the scenario for the analysis of the beaconing schemes. This includes the channel model, user distribution, and beaconing requirements. In Chapter 3, the efficiency bound for plain Aloha is derived. Chapter 4 briefly repeats the description of the coordinated, cell-based beaconing scheme from [4] before deriving the spectral efficiency of this method and presenting results. Chapter 5 explains Advanced Aloha and uses expressions from [6] to calculate its spectral efficiency. Finally, results are compared and conclusions are drawn in Chapter 6.

\section{Beaconing Scenario}

\section{a) Required Performance}

In this paper, beaconing is required to meet a minimum communication range of $r_{\text {com }}$. This means that each node must receive messages at a sufficient rate from all other nodes within a radius of $r_{\text {com }}$ around itself. The rate at which messages are successfully received from any particular neighbor is called sufficient if the time from one message, or update, to the next does not exceed the maximum tolerable update delay, $T_{u}$, with probability $\xi$. Furthermore, each message is required to carry $L$ bits of information.

While the derivation of results in the following chapters is kept general, the following values are used in all example calculations. The communication range is set to $150 \mathrm{nmi}$. This corresponds to the largest fixed-range transmission volume defined for broadcast in the COCR [1]. For $\xi$, a value of 0.95 is assumed, also in line with [1]. Lastly, $L=320$ is used. This exceeds the 34 bytes assumed for surveillance beaconing in [1], in order to provide additional capacity for future applications, e.g., the exchange of control information in an aeronautical MANET as presented in [2]. Note that none of the results to be presented requires an explicit assumption for $T_{u}$.

\section{b) Channel Model, Received Power Levels}

For MANET beaconing, the power imbalances between messages at a receiver have to be taken into account. As there are many receivers for each message, it is not possible to control transmit powers such that all messages arriving at a receiver have about the same power. Therefore, a constant transmit power is assumed for all nodes. Furthermore, the transmission channel is modeled by a channel loss exponent of $\alpha$, and propagation is limited by the radio horizon, denoted by $r_{h}$. A message from a transmitter at a distance $r$ will thus be received with the following power:

$$
P_{r x}(r)= \begin{cases}P_{r x}\left(r_{r e f}\right)\left(\frac{r_{r e f}}{r}\right)^{\alpha}, & \text { if } r \leq r_{h}, \\ 0, & \text { if } r>r_{h} .\end{cases}
$$

In Eqn. (1), $P_{r x}\left(r_{\text {ref }}\right)$ denotes the known received power for some reference distance $r_{\text {ref }}$ to the transmitter. As in this paper, the focus lies on aeronautical scenarios, free space propagation, i.e. $\alpha=2$, is assumed for all numerical examples and figures to be presented. For $r_{h}$, a value of $500 \mathrm{nmi}$ is used. According to an effective earth radius factor of $4 / 3$, this is the radio horizon between two antennas 41300 feet above the ground [7]. Note that for reasons of simplicity, the same altitude, and hence, radio horizon, is assumed for all aircraft. This is a worst-case assumption, as in reality, interference from transmitters at lower altitudes would have a range of less than $r_{h}$.

\section{c) Node Distribution}

The distribution of nodes is modeled by a twodimensional Poisson Point Process in this paper. Such a model is for example also used in [8]. The node density is denoted by $\rho$, which means that the number of nodes in an area of size $A$ is Poissondistributed with mean $\rho A$. Looking at the twodimensional case is in line with our worst-case assumption of equal altitudes from the last paragraph.

Using the parameters discussed so far, an important quantity for the following chapters shall be explained. First, define the nominal per-node data rate as the minimum rate which would be sufficient to achieve $\xi=1$ if there were no message losses. This rate is $L / T_{u}$. Now, assume that a total bandwidth $W$ is available for beaconing. Then, the nominal, 
average data rate generated by all nodes, per area and bandwidth, can be expressed as

$$
\Gamma=\rho \frac{L}{T_{u} W} .
$$

Multiplying this with the area from which a node has to receive information, the nominal, average, bandwidth-normalized traffic "seen" by a receiver is obtained as $\Gamma \pi r_{\text {com }}^{2}$. This will also be called the nominal spectral efficiency at a receiver. It is the nominal rate per bandwidth, at which a node receives information, ignoring message loss. As long as the update delay is met with probability $\xi$, message loss can be ignored in beaconing. In the following chapters, the objective will be to find the maximum $\Gamma \pi r_{\text {com }}^{2}$ for which the requirements can still be fulfilled. This is equivalent to maximizing the user density which can be supported within a given bandwidth. Table 1 summarizes the most important parameters of the beaconing scenarios, and, where appropriate, gives the values which will be assumed for numerical examples in this paper.

Table 1. Parameters and values used for examples in this paper

\begin{tabular}{|l|l|l|}
\hline Prm. & Value & Description \\
\hline$r_{\text {com }}$ & $150 \mathrm{nmi}$ & $\begin{array}{l}\text { Required communication } \\
\text { range }\end{array}$ \\
\hline$r_{h}$ & $500 \mathrm{nmi}$ & Radio horizon \\
\hline$\alpha$ & 2 & Channel loss exponent \\
\hline$L$ & 320 & Bit per message \\
\hline$T_{u}$ & - & Required update delay \\
\hline$\xi$ & 0.95 & $\begin{array}{l}\text { Required probability of not } \\
\text { exceeding update delay }\end{array}$ \\
\hline$W$ & - & $\begin{array}{l}\text { Total beaconing bandwidth } \\
\text { Nominal traffic per } \\
\text { bandwidth and area }\end{array}$ \\
\hline$\Gamma$ & - & $\begin{array}{l}\text { Nominal spectral efficien- } \\
\text { cy at a receiver }\end{array}$ \\
\hline$\Gamma \pi r_{c o m}^{2}$ & - &
\end{tabular}

\section{Plain Aloha}

\section{a) Upper Bound}

As first beaconing scheme, plain Aloha shall be investigated. Herein, a receiver model similar to [9] shall be considered for plain Aloha, where receivers are capable of message capture, but not of simultaneously receiving multiple packets. We begin with the notion of dominant interference. An interfering message overlapping with a desired message is called dominant, if it is by itself strong enough to prevent correct reception of the desired message [8], [10]. With this definition, a lower bound on the message error probability of beaconing may be derived by calculating the probability that a message does not suffer from dominant interference. The following analysis is based on this lower bound for plain Aloha, which leads to an upper bound on the possible spectral efficiency.

At first, consider a desired message of the weakest possible received power, i.e., from a transmitter at distance $r_{\text {com }}$. As in Aloha, the error probability will be a monotonically increasing function of the desired signal power, it is sufficient for our purposes to look at this case. Next, assume that an interfering message arrives with time offset $t$ to the desired message starting point, $t \in\left[-T_{m}, T_{m}\right]$, with the duration of one message denoted by $T_{m}$. Then, the interfering message is dominant if it originates from a certain area around the receiver, i.e., if the interfering transmitter is too close to the receiver. The size of this area for dominant interferers shall be called $A_{\text {dom }}\left(t, r_{\text {com }}\right)$. Integrating over all possible time offsets, the volume $V$ of the region in time and space from which dominant interference originates may be expressed as

$$
V\left(r_{\text {com }}\right)=\int_{-T_{m}}^{T_{m}} A_{\text {dom }}\left(t, r_{\text {com }}\right) d t .
$$

It can now be shown that the number of dominant interferers is a Poisson-distributed random variable, under the Poisson assumption about node distribution used herein. The mean, called $M_{\text {dom }}$, can be obtained by multiplying the average number of messages per time and area with $V$. If nodes transmit at the $k$ times increased nominal beaconing rate, i.e., one message every $T_{u} / k$, then

$$
M_{\text {dom }}\left(r_{\text {com }}\right)=k \frac{\Gamma}{L} W V\left(r_{\text {com }}\right) .
$$

Note that only integer $k$ makes sense, as for, e.g., $k=2.5$, the update delay requirement would be 
violated by two consecutive message losses, which is not better than with $k=2$. The error probability lower bound can now simply be calculated as the probability that a Poisson-distributed random variable with mean $M_{\text {dom }}$ is larger than one, which yields

$$
p_{\text {error }}\left(r_{\text {com }}\right) \geq 1-\exp \left\{-M_{\text {dom }}\left(r_{\text {com }}\right)\right\} \text {. }
$$

As already indicated above, the update delay will only be violated if $k$ consecutive messages from the same node are lost. To fulfill the update delay percentile requirement,

$$
\left(p_{\text {error }}\left(r_{\text {com }}\right)\right)^{k} \leq 1-\xi
$$

must hold. Inserting (5) into (6) and solving for $M_{\text {dom }}$, we have

$$
M_{\text {dom }} \leq-\ln \left(1-(1-\xi)^{1 / k}\right) .
$$

Now, it is appropriate to introduce the coding and modulation rate of a single message, called $a$. It gives the bitrate of a single message, normalized to the bandwidth used by that message, which is the whole beaconing bandwidth for Aloha:

$$
a=\frac{L}{T_{m} W}
$$

Solving (8) for $W$ and inserting this into (4) leads to a representation, which is independent of $L$ and $W$. Inserting this into (7) and solving for $\Gamma \pi r_{c o m}^{2}$ finally yields

$$
\Gamma \pi r_{\text {com }}^{2} \leq g(k) f\left(r_{\text {com }}, a\right),
$$

with

$$
g(k)=-\frac{\ln \left(1-(1-\xi)^{1 / k}\right)}{k},
$$

and

$$
f\left(r_{\text {com }}, a\right)=\frac{a T_{m} \pi r_{c o m}^{2}}{V\left(r_{\text {com }}, a\right)} .
$$

Note that in general, $V$ will depend on $a$, due to its influence on robustness against interference.
What remains to be done, is to establish $A_{\text {dom }}$. This requires a threshold to decide whether an interfering message is dominant. Approximating interference as Gaussian noise, the Shannon capacity of an Additive White Gaussian Noise (AWGN) channel can be used to obtain a threshold for the tolerable Signal to Interference and Noise Ratio (SINR), which yields

$$
a \leq \log _{2}(1+\mathrm{SINR}) .
$$

This threshold is also used in [6] and [8]. Note that according to (1), the instantaneous Signal to Interference Ratio (SIR) for a single, interfering transmitter at distance $r_{i} \leq r_{h}$ is $\left(r_{i} / r_{\text {com }}\right)^{\alpha}$. Again, $r_{\text {com }}$ is assumed for the distance to the transmitter of the desired message. To calculate SINR, however, energies shall be considered, not instantaneous powers. Then, the interference energy contribution of an overlapping message is proportional to its degree of overlap. Denoting the Signal to Noise Ratio by $\operatorname{SNR}\left(r_{\text {com }}\right)$, we obtain for a single interfering message with a time offset of $t$ to the desired message, and distances as mentioned before:

$$
\operatorname{SINR}=\left(\left(\frac{r_{\text {com }}}{r_{i}}\right)^{\alpha}\left(1-\frac{|t|}{T_{m}}\right)+\operatorname{SNR}\left(r_{\text {com }}\right)^{-1}\right)^{-1} .
$$

Inserting (13) into (12) and solving for $r_{i}$ yields:

$$
r_{i}=r_{\text {com }}\left(\frac{\left(1-|t| / T_{m}\right)\left(2^{a}-1\right) \operatorname{SNR}\left(r_{\text {com }}\right)}{\operatorname{SNR}\left(r_{\text {com }}\right)-\left(2^{a}-1\right)}\right)^{1 / \alpha}
$$

Observe that dominant interfering messages for time offset $t$ are those from a disk around the receiver with radius $r_{i}$, as long as $r_{\text {com }} \leq r_{i} \leq r_{h}$. Obviously, when $r_{i}>r_{h}$, the disk radius will be $r_{h}$. As the receiver considered herein cannot process multiple messages at the same time, the best-case assumption is that in the presence of a stronger message, the receiver will abandon the weaker message and capture the stronger one, given that the SINR for the stronger message is sufficient. This means that the disk radius cannot be smaller than $r_{\text {com }}$. As a result,

$$
A_{\text {dom }}\left(t, r_{\text {com }}, a\right)=\pi r_{I}\left(t, r_{\text {com }}, a\right)^{2}
$$


is obtained, using the definition

$$
r_{I}\left(t, r_{\text {com }}, a\right):=\min \left[\max \left[r_{i}, r_{\text {com }}\right], r_{h}\right] .
$$

Maximizing the right-hand size of (9) over $k$ and $a$, for fixed $r_{\text {com }}$, results in the desired upper bound on the nominal spectral efficiency at a receiver. Unfortunately, (16) leads to an unwieldy, piecewise definition of $f\left(r_{\text {com }}, a\right)$. For the results, this function will be maximized numerically. Interestingly, however, $g(k)$ depends only on $k$, and can be maximized independently.

\section{b) Results}

In a first step, $g(k)$ can be maximized for $\xi=0.95$. This yields an optimal value $k_{\text {opt }}=4$, with $g\left(k_{\text {opt }}\right) \approx 0.16$. Using this, $g\left(k_{\text {opt }}\right) f\left(r_{\text {com }}, a\right)$ can be evaluated as a two-dimensional function in $a$ and $r_{\text {com }}$. An example is displayed in Figure 1, using the parameters from Table 1, and an SNR of $10 \mathrm{~dB}$ at $r_{\text {com }}=150 \mathrm{nmi}$. SNR for other values of $r_{\text {com }}$ is calculated according to (1), assuming a constant transmit power. Note that for the figure, $r_{\text {com }}$ has been normalized to $r_{h}$, and $r_{\text {com }} / r_{h}=0.3$ for $r_{h}=500 \mathrm{nmi}$.

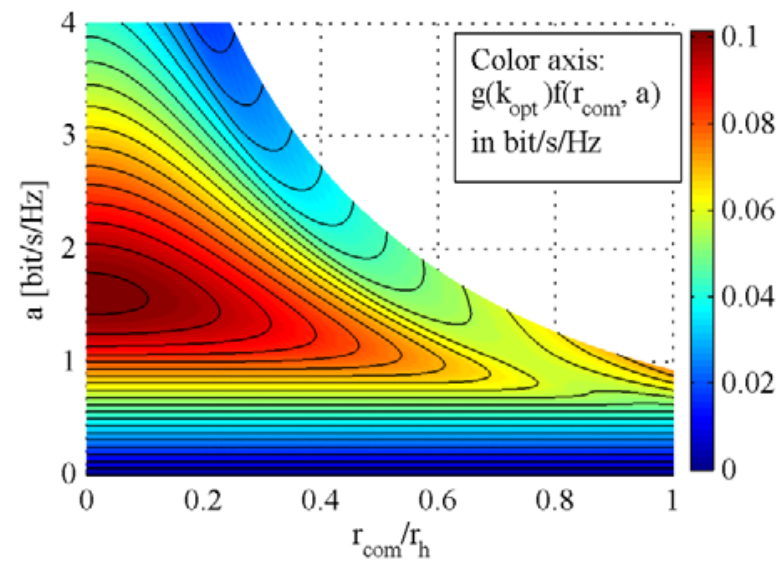

Figure 1. Upper bound for nominal spectral efficiency at a receiver, in $\mathrm{bit} / \mathrm{s} / \mathrm{Hz}$, for SNR of $10 \mathrm{~dB}$ at $r_{\text {com }}=0.3 r_{h}$

Furthermore, note that at a given $r_{\text {com }}$, there is a maximum possible $a$ due to SNR, at which any interference will cause message loss. According to (12), this is $a_{\max }\left(r_{\text {com }}\right)=\log _{2}\left(1+\operatorname{SNR}\left(\mathrm{r}_{\text {com }}\right)\right)$. The border between the colored and white area in Figure 1 represents $a_{\text {max }}\left(r_{\text {com }}\right)$.

Regarding the maximization of $g\left(k_{\text {opt }}\right) f\left(r_{\text {com }}, a\right)$ over $a$, we can see that in the example presented in Figure 1, the optimum $a_{\text {opt }}$ lies between 1 and 2 for small $r_{\text {com }}$. Remarkably, it jumps to $a_{\text {opt }}=a_{\max }$ for, approximately, $r_{\text {com }}>0.74 r_{h}$. Figure 2 shows $g\left(k_{\text {opt }}\right) f\left(r_{\text {com }}, a_{\text {opt }}\right)$ as obtained through numerical maximization. Results are plotted both for the SNR already considered for Figure 1, and for a $10 \mathrm{~dB}$ higher value. Also included are plots evaluated at $a=a_{\max }$. As can be seen, for $r_{\text {com }}=0.3 r_{h}$, the efficiency cannot exceed 0.1 $\mathrm{bit} / \mathrm{sec} / \mathrm{Hz}$ in these examples, and, at least according to the upper bound, gains little from the increase in SNR. This result will be used as a reference value in the following chapters. The communication range at which $a_{\text {opt }}$ becomes $a_{\max }$ can be recognized from Figure 2 as the point where the curves for $a_{o p t}$ and $a_{\max }$ join. From this point onwards, plain Aloha cannot gain anymore from interference robustness through stronger coding, i.e. lower $a$, and hence, one should use $a=a_{\max }$.

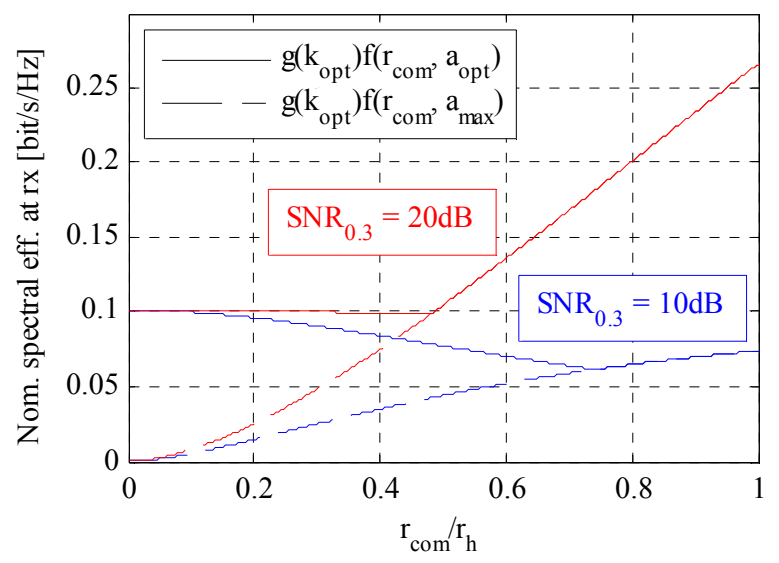

Figure 2. Upper bound for nominal spectral efficiency at a receiver (rx), evaluated at $a_{\text {opt }}$ and $a_{\max }$, for $10 \mathrm{~dB}$ and $20 \mathrm{~dB}$ SNR at $r_{\text {com }}=0.3 r_{h}$ 


\section{Cell-Based Self-Organizing TDMA}

\section{a) Concept}

Now, the coordinated beaconing method discussed in [4] shall be investigated. It has originally been described in [5], and relies on self-organizing TDMA (S-TDMA), which is also used in the VHF Digital Link Mode 4 (VDL4). In S-TDMA, nodes monitor the channel to learn the current occupancy of time slots in the TDMA frame, and then transmit a reservation message to claim for themselves one of the unoccupied slots. This distributed algorithm for the establishment of a TDMA schedule, however, suffers from the hidden terminal problem. It occurs when two nodes which are not in mutual radio range assign to themselves the same slot and their messages collide at a third node in the middle. In order to solve this issue, [5] introduced a pattern of hexagonal cells, and required that nodes transmit on the channel associated to the cell they are located in. Using appropriate cell sizes, this makes sure that all nodes on the same channel are within range of each other. To limit the number of necessary channels, a cellular reuse pattern is employed.

In [4], this concept has been analyzed for the aeronautical scenario. It has been argued that the channels of adjacent cells need substantial isolation between them. One reason for this is that two close aircraft in adjacent cells must be able to transmit on their respective channel and still receive the other aircraft's transmission on the other channel. As a solution, the cells can be assigned non-overlapping sections of the TDMA frame, herein also referred to as TDMA channels, which means that the cells themselves are separated by TDMA [4]. Furthermore, [4] has shown that one main issue for the cell-based beaconing scheme in the aeronautical scenario are the excessive guard times due to the huge propagation delays. However, the loss of efficiency through guard times is increased by the separation of cells via TDMA. In a concurrent access scheme, e.g., when the total system bandwidth is split up via Frequency Division Multiple Access (FDMA), messages take longer, whereby the impact of guard times is reduced. Another advantage of longer message durations is the increase in received energy per bit, when transmit powers are assumed to be constant. Therefore, [4] introduced the nesting of an inner reuse pattern into an outer one, the outer one associated with TDMA, and the inner one with another access scheme, for which FDMA will be assumed in this paper. This way, close cells can be separated by TDMA, while cells at intermediate distance can use the same time slots, but on different frequencies. In the following, the size of the TDMA reuse pattern is denoted by $N_{T}$, while $N_{F}$ refers to the size of the FDMA pattern. Pattern sizes are identical to the respective number of TDMA or FDMA channels. The total number of cells then is [4]

$$
N_{\text {cell }}=N_{T} N_{F} .
$$

An example with $N_{T}=N_{F}=4$ is depicted in Figure 3. TDMA and FDMA channel numbers are apparent from the labels $(\mathrm{T} / \mathrm{F})$ on the cells. The TDMA pattern is additionally marked by colors. The bold lines delimit a cluster, i.e., a complete set of all 16 cell types.

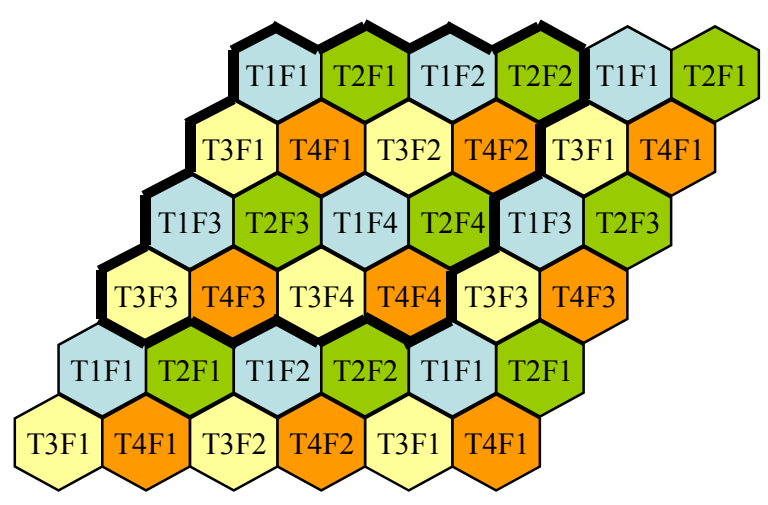

\section{Figure 3. Nesting of FDMA reuse pattern (F) into outer, TDMA reuse pattern ( $T$ )}

For the following analysis, we shortly recall some fundamentals of cellular reuse patterns, for a more detailed explanation, see [7]. At first, only certain integers are valid reuse pattern sizes. A size $N$ is valid if, and only if, two integers $i>0$ and $j \geq 0$ exist such that

$$
N=i^{2}+i j+j^{2} .
$$

If this holds for $N_{T}$ and $N_{F}$, with potentially different $i$ and $j$, then it can be shown that the product, $N_{\text {cell }}$, fulfills the requirement as well. Furthermore, let $R_{\text {cell }}$ denote the cell radius, i.e., the distance from the center of the hexagon to any of its 
vertices. Then the closest distance between two cells of the same channel is

$$
D=R_{\text {cell }} \sqrt{3 N} .
$$

Note that $r_{\text {com }} / 2$ is the largest $R_{\text {cell }}$ considered herein, as for larger $R_{\text {cell }}$, the cell of a node might not be completely within its communication range.

For aeronautical surveillance beaconing, one important drawback of the coordinated cell scheme is its dependence on an accurate, common time reference available to all nodes due to the use of TDMA. Typically, a satellite navigation system would provide this reference. However, an aeronautical surveillance system is required to work even in the event of failure of satellite navigation systems, when aircraft use alternative navigation aids to determine their position. Systems like VDL4 have been designed with fallback solutions for this case. Yet, those normally involve significant degradation of system capabilities in case of prolonged time reference outage, or rely on ground stations as a backup source for the reference time. Another issue to consider is that due to terrain, aircraft on or close to the ground might not be able to receive messages from all others within the cell. This might require the use of smaller cells than desired.

\section{b) Spectral Efficiency}

The basic idea for determining the nominal spectral efficiency at a receiver in the coordinated cell scheme is to determine the maximum bandwidthnormalized coding and modulation rate, $a$, at which messages can be transmitted. Note that in the case of $N_{F}>1, a$ has to be calculated with respect to the bandwidth of a single channel. Again, (12) shall be used to obtain $a$ from the worst-case SINR. Let $\mathrm{SIR}_{w c}$ denote the worst-case signal-to-interference ratio for a given cell scheme. Then, the worst-case SINR can be obtained as

$$
\operatorname{SINR}_{w c}=\left(\operatorname{SIR}_{w c}^{-1}+\left(N_{F} \cdot \operatorname{SNR}\left(\mathrm{r}_{c o m}\right)\right)^{-1}\right)^{-1} .
$$

In (20), the SNR, which is defined with respect to the total system bandwidth, is increased by the number of FDMA channels, because splitting up the total bandwidth decreases the noise bandwidth of the individual channels.
To determine $\mathrm{SIR}_{w c}$ for given $R_{\text {cell }}$ and given $N_{\text {cell }}$, consider a situation as depicted in Figure 4. Cells are approximated by circles of radius $R_{\text {cell }}$. A transmitter is placed on the cell border, i.e., on the circle, at some angle $\varphi$ from, e.g., the x-axis. The receiver is placed at distance $r_{\text {com }}$ from the transmitter, such that the center of the transmitter's cell, the transmitter, and the receiver lie on a straight line with the transmitter between the other two points.

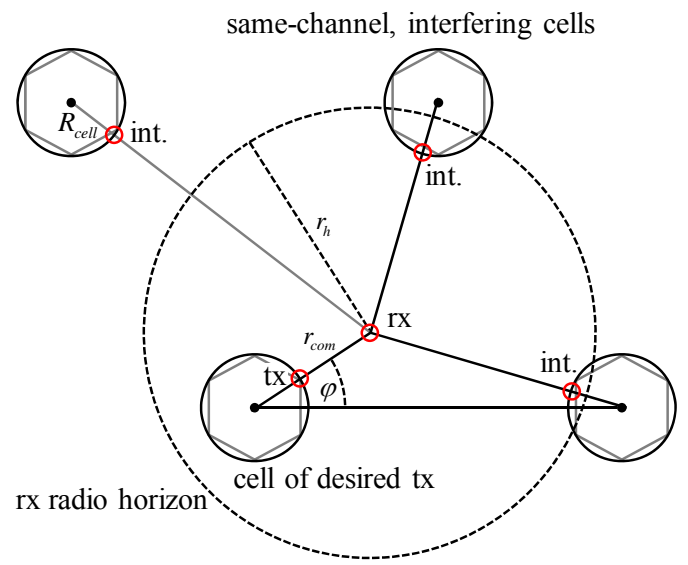

Figure 4. Worst-case interferer (int.) locations in cell pattern with circular approximation

Now, all same-channel cells have to be considered. In the worst-case, there is an interfering transmitter in each of those cells, as close to the receiver as possible. Let $\vec{x}(\varphi)$ be the receiver location, and $\vec{s}$ denote the location of a same-channel cell center. Then, interference may be received from that cell only if $|\vec{x}(\varphi)-\vec{s}|-R_{\text {cell }} \leq r_{h}$, as can be seen from Figure 4. Otherwise, the closest possible transmitter from that cell would not be within the radio horizon of the receiver. Defining $\mathcal{C}(\varphi)$ as the set of all same-channel cells, from which interference can be received, $\operatorname{SIR}(\varphi)$ evaluates to

$$
(\operatorname{SIR}(\varphi))^{-1}=\sum_{\vec{s} \in \mathcal{C}(\varphi)}\left(\frac{r_{\text {com }}}{|\vec{s}-\vec{x}(\varphi)|-R_{\text {cell }}}\right)^{\alpha} .
$$

$\operatorname{SIR}_{w c}$ can be obtained by minimizing $\operatorname{SIR}(\varphi)$ with respect to $\varphi$. What's still needed for the evaluation of (20) is $N_{F}$. To find this, the minimum possible $N_{T}$ has to be established. To fulfill the range 
requirement without having to transmit and receive at the same time, the distance $D_{T}$ between cells of the same TDMA channel must satisfy

$$
D_{T}>2 R_{\text {cell }}+r_{\text {com }},
$$

again using the circular approximation of cells, and worst-case transmitter and receiver locations as in Figure 4. Note that $D_{T}$ can be calculated from $R_{\text {cell }}$ and $N_{T}$ using (19). Now, we can simply check all candidate integers $N_{T, \text { cand }}$ between the minimum possible according to (22), and $N_{\text {cell }}$, whether they are a valid reuse pattern size according to (18), and whether the resulting $N_{F}=N_{\text {cell }} / N_{T, \text { cand }}$ also fulfills this requirement. The minimum $N_{T}$ and corresponding $N_{F}$ fulfilling all conditions may then be selected. $\mathrm{SIR}_{w c}$ and optimized $N_{F}$ allow to determine the maximum possible $a$, using (20) and (12).

Having obtained the maximum $a$, consider the spectral efficiency at a receiver, if only the data traffic from a single cell was received, the complete TDMA section of that cell was occupied, and without any losses due to guard times or coordination overhead. With this denoted by $a_{\text {cell }}$, observe that

$$
a_{\text {cell }}=a / N_{\text {cell }} \text {. }
$$

The relationship can be seen from the fact that a cell can only use that portion of a TDMA frame and that portion of the total bandwidth, which has been allocated to it. However, a receiver can pick up data traffic from more than just one cell. Counting only the nominal traffic, i.e. messages from nodes within $r_{\text {com }}$, we obtain the nominal spectral efficiency at a receiver by multiplying $a_{\text {cell }}$ with the number of cells in range. As the area of a hexagonal cell is $3 \sqrt{3} R_{\text {cell }}^{2} / 2$, this results in

$$
\Gamma \pi r_{\text {com }}^{2}=a_{\text {cell }} \frac{\pi r_{\text {com }}^{2}}{3 \sqrt{3} R_{\text {cell }}^{2} / 2} .
$$

The described process can be repeated for different $N_{\text {cell }}$ and $R_{\text {cell }}$, to find optimal values for these parameters as well. As already indicated, the results neglect guard times, and optimistically assume that each cell is occupied by the maximum possible number of users.

\section{c) Results}

To generate results, $r_{h}, r_{\text {com }}$ and $\alpha$ are set to the values given in Table 1 . Note that $\xi$ is not needed for the cell-based scheme, as coordination limits packet losses to a negligible amount. The SNR has been set to $10 \mathrm{~dB}$ for a distance of $r_{\text {com }}$ between transmitter and receiver. The minimization of $\operatorname{SIR}(\varphi)$ has been performed by sampling $\varphi$ in increments of $1.2^{\circ}$. For a given $R_{\text {cell }}$, all possible pattern sizes have been considered up to a value for which the closest distance between receiver and interfering transmitter is significantly larger than the radio horizon. Figure 5 shows $\Gamma \pi r_{\text {com }}^{2}$ for $R_{\text {cell }}=47.9 \mathrm{nmi}$ and a large range of reuse pattern sizes. The latter can be divided into sizes for which the receiver may suffer from interference, and those which are large enough to make sure that there is no same-channel interference within the radio horizon of a receiver. The results in Figure 5 have been marked accordingly. The smallest pattern size $N_{\text {cell }}$ for which there is no interference turns out to be 81 in this example, with $N_{T}=N_{F}=9$. This is also the value which leads to the largest efficiency. Note that the reason why the results in Figure 5 do not appear on a smooth line is that for some values of $N_{\text {cell }}$, higher values of $N_{F}$ are possible than for others.

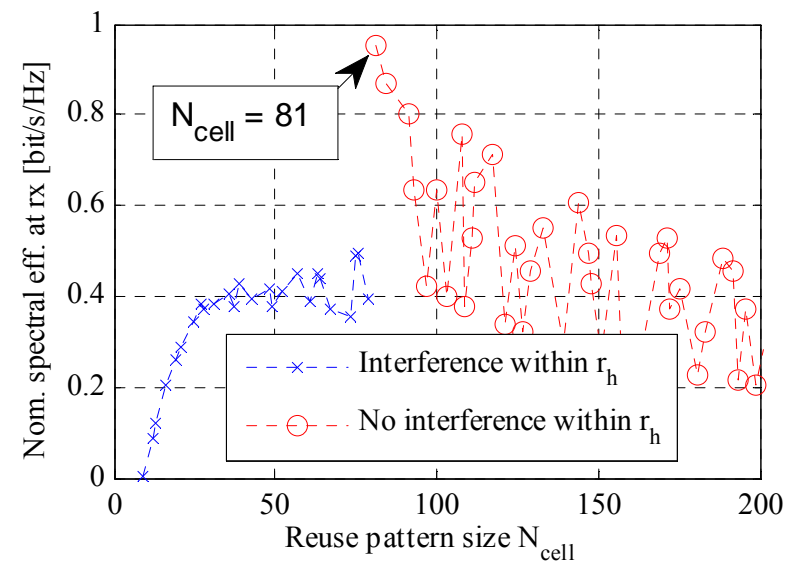

Figure 5. Efficiency of cell-based beaconing with cell radius of $47.9 \mathrm{nmi}$, radio horizon of $500 \mathrm{nmi}$ 
The fact that the efficiency is maximized by a pattern size which is only barely large enough to remove interference from the radio horizon requires further attention. If such a scheme was implemented, an aircraft at a higher altitude than assumed in the cell planning would suffer from message loss through interference. Therefore, an increased radio horizon of $600 \mathrm{nmi}$ is considered for comparison in the results below. Using an effective earth radius factor of $4 / 3$, this radio horizon would only be reached between two aircraft flying at an altitude of about 59500 feet. Further results have been calculated using the parameters explained above, and for $R_{\text {cell }}$ in the ranges of, in nautical miles, 20 to 25,45 to 50 , and 70 to 75 . Within each range, $R_{\text {cell }}$ has been sampled in increments of $0.1 \mathrm{nmi}$, and for each $R_{\text {cell }}$, the optimum $N_{\text {cell }}$ has been determined, which maximizes $\Gamma \pi r_{\text {com }}^{2}$. Subsequently, the maximization may also be performed with respect to $R_{\text {cell }}$. Table 2 lists the resulting maximum $\Gamma \pi r_{c o m}^{2}$, which may be achieved in the given range of $R_{\text {cell }}$. The optimum $R_{\text {cell }}$, for which this maximum is achieved, is given in brackets behind the maximum $\Gamma \pi r_{\text {com }}^{2}$.

Table 2. Efficiency [bit/s/Hz] for small, medium and large cells, with optimum $\mathbf{R}_{\text {cell }}$ in brackets

\begin{tabular}{|l|l|l|}
\hline $\begin{array}{l}\text { Range of } \\
\mathbf{R}_{\text {cell }} \text { [nmi] }\end{array}$ & $\begin{array}{l}\text { Max eff. for } \\
\mathbf{r}_{\mathbf{h}}=\mathbf{5 0 0} \mathbf{~ n m i}\end{array}$ & $\begin{array}{l}\text { Max. eff. for } \\
\mathbf{r}_{\mathbf{h}}=\mathbf{6 0 0} \mathbf{~ n m i}\end{array}$ \\
\hline 20 to 25 & $1.19(21.0 \mathrm{nmi})$ & $0.95(20.6 \mathrm{nmi})$ \\
\hline 45 to 50 & $0.95(47.9 \mathrm{nmi})$ & $0.79(47.0 \mathrm{nmi})$ \\
\hline 70 to 75 & $0.70(70.0 \mathrm{nmi})$ & $0.62(74.1 \mathrm{nmi})$ \\
\hline
\end{tabular}

It is noteworthy that the maximized efficiencies given in Table 2 all rely on interference-free reuse patterns, with $N_{F}$ on the order of 10 . Due to the increase in SNR, this allows values of $a$ as high as 7 in some cases. Whether this is practical, would have to be investigated. It implies another drawback of the coordinated cell scheme, as the employed power amplifiers would have to be much more linear than for a scheme that operates at low $a$ and can use a simple, constant-amplitude modulation alphabet.

The reason for considering several ranges for $R_{\text {cell }}$ is that the results calculated herein assume that cells are occupied by the maximum number of users.
Under this assumption, smaller cells will tend to be more efficient than larger cells. In reality, however, the maximum number of users for which capacity is required in a cell and the average users count would have to be determined from operational constraints and known traffic statistics. The factor between average and maximum would reduce the efficiency, and this factor would typically be worse for smaller average number of users per cell. It is thus not possible to determine the actual optimum $R_{\text {cell }}$ and actual efficiency without making specific assumptions about the maximum and average number of users in a cell. As an example, assume that the number of users in a cell is Poisson-distributed with a mean of 100. From the cumulative distribution function of the Poisson distribution, it can be derived that if the maximum number of users the cell can support must not be exceeded with probability $10^{-3}$, capacity for 132 users would be required in the cell. If the probability for exceeding the maximum is decreased to $10^{-6}$, capacity for 151 users would be required. Hence, the results would be reduced by a factor of 0.76 or 0.66 , respectively, for a cell with 100 users on average. For small cells and large $r_{h}$ according to Table 2, a maximum of 132 users and an average of 100 users per cell would mean a nominal spectral efficiency at a receiver of $0.72 \mathrm{bit} / \mathrm{s} / \mathrm{Hz}$. Compared to the upper bound for plain Aloha at $r_{\text {com }}=0.3 r_{h}$ and high SNR displayed in Figure 2, this is around 7.2 times higher. Note that the assumed average and maximum user counts might be optimistic, as the average is quite high for such a small cell considering typical peak instantaneous aircraft counts from [1]. Furthermore, the negligence of guard times is optimistic, although [4] argues that guard times may be reduced significantly by making them dynamically depend on the distance from one transmitter to the next.

\section{Advanced Aloha}

It is a well-known fact that the efficiency of Aloha may be improved by Successive Interference Cancellation [11], [12]. In [6], the spectral efficiency of Aloha with SIC has been analyzed, including the optimization of system parameters. As a key result, the efficiency is maximized for as strong as possible coding on the physical layer, i.e. $a \rightarrow 0$. This combined with SIC in the receiver has been termed 
Advanced Aloha [6]. Note that in Advanced Aloha, there are no dominant interferers, as the coding rate is low, and, when processing a message, any stronger, interfering message is already canceled. The results from [6] suggest that by coding and SIC, the error rate of messages from transmitters closer than $r_{\text {com }}$ can be kept arbitrarily low. This is why $\xi$ does not appear in the equations for Advanced Aloha. In addition to the parameters already introduced in this paper, [6] considers the required SINR for synchronization, $Z$. The notion is that each message contains a synchronization sequence known to the receiver, which can be used to detect messages through correlation. $Z$ is the minimum SINR at the output of the synchronization correlator for which messages can still be detected. Furthermore, [6] considers the ratio of received energy per bit to the power spectral density of thermal noise, $E_{b} / N_{0}$. Herein however, the transmit power of a node is assumed constant, which means that the transmitted energy per bit is proportional to $1 / a$. Hence, $E_{b} / N_{0}$ will become large for small $a$. Therefore, Advanced Aloha results without thermal noise are considered herein. For free space propagation, we obtain from [6, Eqns. (13)-(19)]

$$
r_{t h}=r_{h} \exp (-1 / 2)
$$

and

$$
\begin{aligned}
& \Gamma \pi r_{\text {com }}^{2}= \\
& \begin{cases}L\left(2 \ln \left(\frac{r_{h}}{r_{\text {com }}}\right)(L \ln (2)+Z)\right)^{-1}, & \text { if } r_{\text {com }} \leq r_{\text {th }}, \\
L(L \ln (2)+Z)^{-1}\left(\frac{r_{\text {com }}}{r_{\text {th }}}\right)^{2}, & \text { if } r_{\text {com }}>r_{t h} .\end{cases}
\end{aligned}
$$

The distance $r_{\text {th }}$ given by (25) is the threshold radius. For $r_{\text {com }}$ larger than this value, the allowable user density does not decrease with distance anymore, which means that $\Gamma \pi r_{c o m}^{2}$ becomes proportional to $r_{\text {com }}^{2}$, cf. (26).

To plot results, a value for $Z$ is needed in addition to $L=320$ from Table 1 . To get a reasonable assumption, consider the false alarm and missed detection probabilities in Gaussian noise, which is again used to approximate interference. For example, with a detection threshold achieving a false alarm rate of $10^{-6}, Z=15.34 \mathrm{~dB}$ is necessary to get a missed detection probability of $10^{-3}$. The latter should neither be a problem for the Advanced Aloha receiver, which will only very rarely suffer from uncanceled interference due to missed detection events, nor for the requirement of $\xi=0.95$.

Figure 6 plots $\Gamma \pi r_{\text {com }}^{2}$ for Advanced Aloha with the parameters explained above. The upper bound for plain Aloha with a SNR of $20 \mathrm{~dB}$ at $r_{\text {com }}=0.3 r_{h}$, already given in Figure 2, is included as well for comparison. At $r_{\text {com }}=0.3 r_{h}$, Advanced Aloha achieves a nominal spectral efficiency at the receiver of $0.52 \mathrm{bit} / \mathrm{s} / \mathrm{Hz}$. This is approximately 5.2 times higher than the plain Aloha upper bound.

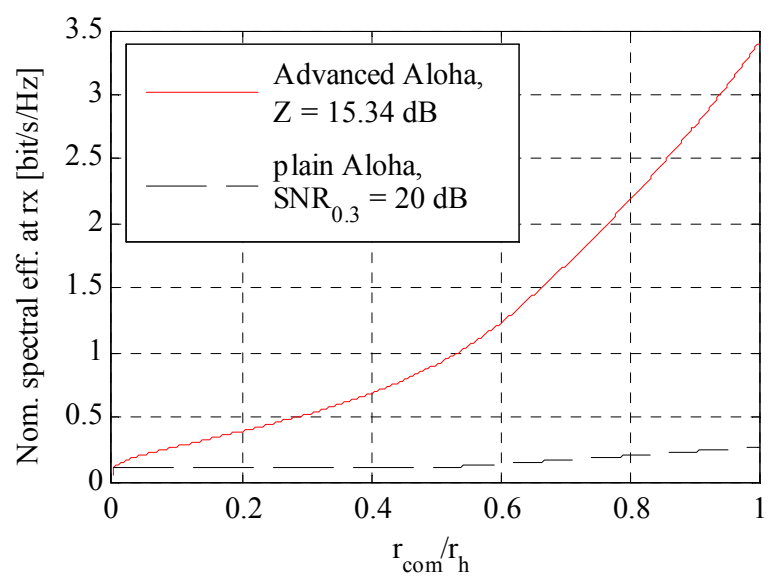

Figure 6. Nominal spectral eff. at rx for Advanced Aloha vs. plain Aloha upper bound (cf. Figure 2)

\section{Conclusion}

In this paper, it could be shown that two advanced beaconing schemes, Advanced Aloha, and cell-based self-organizing TDMA, significantly outperform plain Aloha with respect to the nominal spectral efficiency at a receiver in the aeronautical scenario considered herein. Comparing results for the cell-based approach and Advanced Aloha at $r_{\text {com }}=0.3 r_{h}$, the cell-based approach could be around 38 percent more bandwidth-efficient than Advanced Aloha. This relies on the efficiency given in Table 2 for small cells and large radio horizon, reduced by a 
factor of 0.76 according to the hypothetical example of 100 users per cell on average and 132 maximum, as discussed at the end of Chapter 4. However, recall that this result for the coordinated scheme also relies on optimistic assumptions about the user density and guard times. Even if those hold, and said advantages over Advanced Aloha could be realized, the latter might still be better suited to aeronautical surveillance beaconing. It is questionable whether the moderately higher efficiency justifies accepting the drawbacks of the coordinated cell scheme discussed in Chapter 4, especially the dependence on an accurate time reference. As long as a coordinated beaconing scheme is not much more efficient than a good, uncoordinated one, the uncoordinated option should be used, as it does not require a common time reference, and does not suffer from guard times.

\section{References}

[1] Eurocontrol/FAA, 2007, "Communications Operating Concept and Requirements for the Future Radio System," COCR Version 2.0, Technical Report.

[2] D. Medina et al., 2011, "A Geographic Routing Strategy for North Atlantic In-Flight Internet Access Via Airborne Mesh Networking," IEEE/ACM Transactions on Networking.

[3] N. Abramson, 1970, "The ALOHA System Another Alternative for Computer Communications," AFIPS Conference Proceedings, Vol. 37, pp.281-285.

[4] M. Walter, N. Franzen, M. Schnell, 2010, "New concepts for a decentralized, self-organizing air-to-air radio link," 29th Digital Avionics Systems Conference (DASC), Salt Lake City, UT.

[5] C. Rico Garcia, A. Lehner, T. Strang, 2008, "COMB: Cell Based Orientation Aware MANET Broadcast MAC Layer", IEEE Global Communications Conference 2008 (GLOBECOM 2008), New Orleans, LA.
[6] N. Franzen, 2011, "Advanced Aloha with SIC for Beaconing in a MANET," 73rd IEEE Vehicular Technology Conference (VTC Spring), Budapest, Hungary.

[7] J. D. Parson, 2000, "The Mobile Radio Propagation Channel," 2nd ed., John Wiley \& Sons.

[8] S. Weber, J. G. Andrews, N. Jindal, 2010, “An Overview of the Transmission Capacity of Wireless Networks", IEEE Transactions on Communications, vol. 58 , no. 12 , pp. $3593-3604$.

[9] R. Nelson, L. Kleinrock, 1984, "The Spatial Capacity of a Slotted Aloha Multihop Packet Radio Network with Capture," IEEE Transactions on Communications, vol. 32, no. 6, pp. 684-694.

[10] J. G. Andrews, S. Weber, M. Haenggi, 2007, "Ad Hoc Networks: To Spread or Not to Spread?" IEEE Communications Magazine, vol. 45, no. 12, pp. 84-91.

[11] A. J. Viterbi, 1990, "Very low rate convolution codes for maximum theoretical performance of spread-spectrum multiple-access channels," IEEE Journal on Selected Areas in Communication, vol. 8, no. 4, pp. 641-649.

[12] O. del Rio Herrero and R. D. Gaudenzi, 2009, "A High Efficiency Scheme for Large-Scale Satellite Mobile Messaging Systems," 27th IET and AIAA International Communications Satellite Systems Conference, Edinburgh, UK.

\section{Email Addresses}

N. Franzen: nico.franzen@dlr.de

\section{1st Digital Avionics Systems Conference} October 14-18, 2012 